Fields Institute Communications

Volume 00, 0000

\title{
PBW Bases, Non-Degeneracy Conditions and Applications
}

\author{
Viktor Levandovskyy \\ Center for Computer Algebra \\ Fachbereich Mathematik \\ Universität Kaiserslautern \\ Postfach 3049 D-67653 Germany \\ levandov@mathematik.uni-kl.de
}

\begin{abstract}
We establish an explicit criteria (the vanishing of non-degeneracy conditions) for certain noncommutative algebras to have Poincaré-BirkhoffWitt basis. We study theoretical properties of such $G$-algebras, concluding they are in some sense "close to commutative". We use the non-degeneracy conditions for practical study of certain deformations of Weyl algebras, quadratic and diffusion algebras.
\end{abstract}

The famous Poincaré-Birkhoff-Witt (or, shortly, PBW) theorem, which appeared at first for universal enveloping algebras of finite dimensional Lie algebras ([7]), plays an important role in the representation theory as well as in the theory of rings and algebras. Analogous theorem for quantum groups was proved by G. Lusztig and constructively by C. M. Ringel ([6]).

Many authors have proved the PBW theorem for special classes of noncommutative algebras they are dealing with $([17,[18])$. Usually one uses Bergman's Diamond Lemma (4), although it needs some preparations to be done before applying it. We have defined a class of algebras where the question "Does this algebra have a PBW basis?" reduces to a direct computation involving only basic polynomial arithmetic.

In this article, our approach is constructive and consists of three tasks. Firstly, we want to find the necessary and sufficient conditions for a wide class of algebras to have a PBW basis, secondly, to investigate this class for useful properties, and thirdly, to apply the results to the study of certain special types of algebras.

The first part resulted in the non-degeneracy conditions (Theorem 2.3), the second one led us to the $G$ - and $G R$-algebras (3.4) and their properties (Theorem 4.7. 4.8, and the third one - to the notion of $G$-quantization and to the description and classification of $G$-algebras among the quadratic and diffusion algebras.

1991 Mathematics Subject Classification. Primary 68W30, 16W70; Secondary 16Z05, 13P10. partially supported by the CRDF grant UM2-2094 and DFG.

(C)0000 American Mathematical Society 
In this article we have simplified many proofs of known results and unified different notations. As far as we know, no article before this one featured a complete treatment of the problems, arising in connection with PBW bases.

We are deeply grateful to Prof. Yu. Drozd and Prof. E. Green for their hints, critics and suggestions that contributed greatly to this article. We also would like to thank Dr. H. Schönemann, Dr. W. Seiler and O. Khomenko for fruitful discussions concerning this article.

\section{Gröbner bases on tensor algebras}

Let $\mathbb{K}$ be a field and $T=\mathbb{K}\left\langle x_{1}, \ldots, x_{n}\right\rangle$ a tensor algebra. We will omit the tensor product sign while writing multiplication and we will mean by an ideal a two-sided ideal, whenever no confusion is possible.

We say that monomials in $T$ are elements from the set of all words

$$
\operatorname{Mon}(T)=\left\{x_{i_{1}}^{\alpha_{1}} x_{i_{2}}^{\alpha_{2}} \ldots x_{i_{m}}^{\alpha_{m}} \mid 1 \leq i_{1}, i_{2}, \ldots, i_{m} \leq n, \alpha_{k} \geq 0\right\} .
$$

A set of standard monomials which we will need later is defined as

$$
\operatorname{Mon}_{S}(T)=\left\{x_{i_{1}}^{\alpha_{1}} x_{i_{2}}^{\alpha_{2}} \ldots x_{i_{m}}^{\alpha_{m}} \mid 1 \leq i_{1}<i_{2}<\ldots<i_{m} \leq n, \alpha_{k} \geq 0\right\} .
$$

Note, that a natural $\mathbb{K}$-basis of a commutative polynomial ring $\mathbb{K}\left[x_{1}, \ldots, x_{n}\right]$ is exactly the PBW basis. Therefore, algebras which are noetherian domains with PBW basis are in this sense "close to commutative".

Now we will present the short account of the Gröbner bases theory on tensor algebras. It was first Teo Mora, who considered a unified Gröbner bases framework for commutative and noncommutative algebras (23]), which has been recently exploited also by $\mathrm{Li}([16)$. We follow this approach partially, using in addition the articles 13 and 14 and writing in the spirit of 12 thus keeping almost the same notations with the 21].

Definition 1.1 We call a total ordering < a monomial ordering on $\operatorname{Mon}(T)$ if the following conditions hold:

1. $<$ is a well-ordering on $\operatorname{Mon}(T)$, that is $\forall a \in \operatorname{Mon}(T)$ there exist finitely many $b \in \operatorname{Mon}(T)$ such that $b<a$,

2. $\forall p, q, s, t \in \operatorname{Mon}(T)$, if $s<t$, then $p \cdot s \cdot q<p \cdot t \cdot q$,

3. $\forall p, q, s, t \in \operatorname{Mon}(T)$, if $s=p \cdot t \cdot q$, then $t<s$.

In this work we are dealing with well-orderings only.

Definition 1.2 Any $f \in T \backslash\{0\}$ can be written uniquely as

$f=c \cdot m+f^{\prime}$, where $c \in \mathbb{K}^{*}$ and $m>m^{\prime}$ for any non-zero term $c^{\prime} \cdot m^{\prime}$ of $f^{\prime}$.

We define $\operatorname{lm}(f)=m$, the leading monomial of $f$, $\operatorname{lc}(f)=c$, the leading coefficient of $f$.

For a subset $G \subset T$, define a leading ideal of $G$ to be the two-sided ideal

$$
L(G)=\langle\{\operatorname{lm}(g) \mid g \in G \backslash\{0\}\}\rangle \subseteq T .
$$

Definition 1.3 Let $<$ be a fixed monomial ordering on $T$. We say that a subset $G \subset I$ is a Gröbner basis for $I$ with respect to $<$ if $L(G)=L(I)$.

Although we can work formally with infinite Gröbner bases, in this article we are interested only in finite bases. 
Definition 1.4 Let $m, m^{\prime} \in \operatorname{Mon}(T)$ be two monomials.

We say that $m$ divides $m^{\prime}$ if there exist $p, q \in \operatorname{Mon}(T)$ such that $m^{\prime}=p \cdot m \cdot q$.

The set $G \subseteq T$ is called minimal, if $\forall g_{1}, g_{2} \in G, \operatorname{lm}\left(g_{1}\right)$ does not divide $\operatorname{lm}\left(g_{2}\right)$ and vice versa.

Definition 1.5 Let $\mathcal{G}$ be the set of all finite and ordered subsets of $T$.

A map NF : $T \times \mathcal{G} \rightarrow T,(f, G) \mapsto \mathrm{NF}(f \mid G)$ is called a normal form on $T$ if

(i) $\mathrm{NF}(f \mid G) \neq 0 \Rightarrow \operatorname{lm}(\mathrm{NF}(f \mid G)) \notin L(G)$, and

(ii) $f-\operatorname{NF}(f \mid G) \in\langle G\rangle$, for all $f \in T$ and $G \in \mathcal{G}$.

Algorithm 1.6 Let $<$ be a well-ordering on $T$.

$\mathrm{NF}(f \mid G)$

Input: $f \in T, G \in \mathcal{G}$.

Output: $h \in T$, a normal form of $f$ with respect to $G$.

- $h=f$;

- While $\left(h \neq 0\right.$ and $G_{h}=\{g \in G \mid \operatorname{lm}(g)$ divides $\left.\operatorname{lm}(h)\} \neq \emptyset\right)$

choose any $g \in G_{h}$;

compute $l=l(g), r=r(g) \in \operatorname{Mon}(T)$ such that $\operatorname{lm}(h)=l \cdot \operatorname{lm}(g) \cdot r$;

$h=h-\frac{l c(h)}{l c(g)} \cdot l \cdot g \cdot r ;$

- RETURN $h$;

Proof We shall prove termination and correctness of the algorithm.

We see that each specific choice of "any" in the algorithm may give us a different normal form function. Let $h_{0}:=f$, and in the $i$-th step of the WhILE loop we compute $h_{i}$. Since $\operatorname{lm}\left(h_{i}\right)<\operatorname{lm}\left(h_{i-1}\right)$ by the construction, we obtain a set $\left\{\operatorname{lm}\left(h_{i}\right)\right\}$ of leading monomials of $h_{i}$, where $\forall i h_{i+1}$ has strictly smaller leading monomial than $h_{i}$. Since $<$ is a well-ordering, this set has a minimum, hence the algorithm terminates.

Suppose this minimum is reached at the step $m$. Let $h=h_{m}$ and $l_{i}, r_{i}$ are monomials, corresponding to $g_{i} \in G$ in the algorithm. Making back substitutions, we obtain the following expression

$$
h=f-\sum_{i=1}^{m-1} l_{i} g_{i} r_{i}
$$

satisfying $\operatorname{lm}(f)=\operatorname{lm}\left(l_{1} g_{1} r_{1}\right)>\operatorname{lm}\left(l_{i} g_{i} r_{i}\right)>\operatorname{lm}\left(h_{m}\right)$.

Moreover, by the construction $\operatorname{lm}(h) \notin L(G)$. This proves correctness, independently of the specific choice of "any" in the WHILE loop.

Definition 1.7 Let $f, g \in T$. Suppose that there are $p, q \in \operatorname{Mon}(T)$ such that

1. $\operatorname{lm}(f) q=p \operatorname{lm}(g)$

2. $\operatorname{lm}(f)$ does not divide $p$ and $\operatorname{lm}(g)$ does not divide $q$.

Then the overlap relation of $f, g$ by $p, q$ is defined as

$$
o(f, g, p, q)=\frac{1}{\operatorname{lc}(f)} f q-\frac{1}{\operatorname{lc}(g)} p g .
$$

We see that $\operatorname{lm}(o(f, g, p, q))<\operatorname{lm}(f) q=p \operatorname{lm}(g)$, hence, overlap relation is a generalization of the notion of $s$-polynomial from the commutative theory (cf. [12]).

The next theorem is a slightly reformulated Termination theorem from [14. 
Theorem 1.8 Let $<$ be an well-ordering on $T$ and $G$ be a finite set of polynomials from $T$. If for every overlap relation with $g_{1}, g_{2} \in G$

$$
\mathrm{NF}\left(o\left(g_{1}, g_{2}, p, q\right) \mid G\right)=0,
$$

then $G$ is a Gröbner basis for $\langle G\rangle$.

\section{Non-degeneracy conditions on tensor algebra and PBW theorem}

Again, let $T=\mathbb{K}\left\langle x_{1}, \ldots, x_{n}\right\rangle$ be a tensor algebra.

For fixed $n$, define the set of indices $\mathcal{U}_{m}:=\left\{\left(i_{1}, \ldots, i_{m}\right) \mid 1 \leq i_{1}<\ldots<i_{m} \leq n\right\}$.

Suppose there are two sets $C=\left\{c_{i j}\right\} \subset \mathbb{K}^{*}$ and $D=\left\{d_{i j}\right\} \subset T$, where $(i, j) \in \mathcal{U}_{2}$. We construct a set $F=\left\{f_{j i} \mid(i, j) \in \mathcal{U}_{2}\right\}$, where

$$
f_{j i}=x_{j} x_{i}-c_{i j} \cdot x_{i} x_{j}-d_{i j} .
$$

We require the existence of a well-ordering $<$ on $T$, such that $\operatorname{lm}\left(f_{j i}\right)=x_{j} x_{i}$ and $\operatorname{lm}\left(d_{i j}\right)<x_{i} x_{j}$. Moreover, we assume that polynomials $d_{i j}$ are already given in terms of standard monomials (if in some $d_{i j}$ there is a nonstandard monomial with coefficient $c \cdot m$, then $m$ is divisible by some $\operatorname{lm}\left(f_{k l}\right)$, hence we replace every such $m$ with $\operatorname{NF}(m \mid F)$, and iterate this procedure until we get a polynomial in standard monomials. It terminates since $<$ is a well-ordering). Then we construct the two-sided ideal $I=\langle F\rangle \subset T$.

For $(i, j, k) \in \mathcal{U}_{3}$ define the non-degeneracy condition for $(i, j, k)$ to be $\mathcal{N D C}_{i j k}=c_{i k} c_{j k} \cdot d_{i j} x_{k}-x_{k} d_{i j}+c_{j k} \cdot x_{j} d_{i k}-c_{i j} \cdot d_{i k} x_{j}+d_{j k} x_{i}-c_{i j} c_{i k} \cdot x_{i} d_{j k}$.

Lemma 2.1 $F$ is a Gröbner basis for $I$ with respect to $<$ if and only if $\forall 1 \leq i<j<k \leq n \quad \mathrm{NF}\left(\mathcal{N D C}_{i j k} \mid F\right)=0$.

Proof We will compute Gröbner basis of $I$ symbolically, but as explicitly as we can. Following the theorem 1.8, we have to consider all the possible overlaps of elements from $F$. It's straightforward, that the only nonzero overlaps can occur for the set of pairs $\left\{\left(f_{j i}, f_{k j}\right) \mid(i, j, k) \in \mathcal{U}_{3}\right\}$. Computing the overlap relation of $\left(f_{j i}, f_{k j}\right)$ for fixed $(i, j, k) \in \mathcal{U}_{3}$, we get

$$
\begin{gathered}
o_{1}=x_{k} x_{j} x_{i}-c_{i j} x_{k} x_{i} x_{j}-x_{k} d_{i j}-x_{k} x_{j} x_{i}+c_{j k} x_{j} x_{i}+d_{j k} x_{i}= \\
=-c_{i j} x_{k} x_{i} x_{j}+c_{j k} x_{j} x_{k} x_{i}-x_{k} d_{i j}+d_{j k} x_{i} .
\end{gathered}
$$

The $o_{1}$ can be reduced with $f_{k j}$ to

$$
o_{2}=c_{j k} x_{j} x_{k} x_{i}-c_{i j} c_{i k} x_{i} x_{k} x_{j}-c_{i j} d_{i k} x_{j}-x_{k} d_{i j}+d_{j k} x_{i},
$$

where $o_{2}$ could be further reduced with $f_{k i}$ to

$$
o_{3}=c_{j k} c_{i k} x_{j} x_{i} x_{k}-c_{i j} c_{i k} x_{i} x_{k} x_{j}-c_{i j} d_{i k} x_{j}-x_{k} d_{i j}+d_{j k} x_{i}+c_{j k} x_{j} d_{i k} .
$$

On its own, we reduce $o_{3}$ with $f_{j i}$ to

$o_{4}=-c_{i j} c_{i k} x_{i} x_{k} x_{j}+c_{j k} c_{i k} c_{i j} x_{i} x_{j} x_{k}+c_{j k} c_{i k} d_{i j} x_{k}-c_{i j} d_{i k} x_{j}-x_{k} d_{i j}+d_{j k} x_{i}+c_{j k} x_{j} d_{i k}$,

and, respectively, $f_{k j}$ finishes the reduction of $o_{4}$ :

$$
o_{5}=c_{j k} c_{i k} d_{i j} x_{k}-x_{k} d_{i j}+c_{j k} x_{j} d_{i k}-c_{i j} d_{i k} x_{j}+d_{j k} x_{i}-c_{i j} c_{j k} x_{i} d_{j k} .
$$

As we see, $o_{5}=\mathcal{N} \mathcal{D} \mathcal{C}_{i j k}$, and $o_{5}$ cannot be further reduced with the elements of $F$ without the more specific information on $\left\{d_{i j}\right\}$. So, if $\operatorname{NF}\left(\mathcal{N D \mathcal { C }} \mathcal{C}_{i j k} \mid F\right) \neq 0$, $F$ is not a Gröbner basis of $I$. Hence the claim.

Lemma 2.2 With the same notation as before, a $\mathbb{K}$-algebra $A=T / I$ has a PBW basis if and only if $F$ is a Gröbner basis for $I$ with respect to $<$. 
Proof If $F$ is a Gröbner basis for $I$ with respect to $<$, the underlying $\mathbb{K}^{-}$ vector space of $A$ is generated by $\left\{m \in \operatorname{Mon}(T) \mid \operatorname{lm}\left(f_{j i}\right)\right.$ does not divide $\left.m\right\}$ by the property of Gröbner bases ([13]). We see immediately that this vector space is the set of standard monomials, since no standard monomial is divisible by $\operatorname{lm}\left(f_{j i}\right) \forall j>i$.

Conversely, let $A=T / I$ has a PBW basis. Then we can interpret it as a $\mathbb{K}$-algebra, generated by $x_{1}, \ldots, x_{n}$ with the multiplication

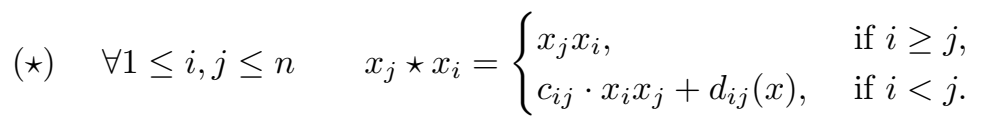

Since $A$ is an associative algebra, $\left(x_{k} \star x_{j}\right) \star x_{i}-x_{k} \star\left(x_{j} \star x_{i}\right)=0 \forall(i, j, k)$. It is easy to see that this holds trivially for all the cases except that when $(i, j, k) \in \mathcal{U}_{3}$, which we analyze. A bit lengthy technical computation in this case delivers

$$
\begin{aligned}
& \left(x_{k} \star x_{j}\right) \star x_{i}-x_{k} \star\left(x_{j} \star x_{i}\right)= \\
& \quad=c_{i k} c_{j k} \cdot d_{i j} x_{k}-x_{k} d_{i j}+c_{j k} \cdot x_{j} d_{i k}-c_{i j} \cdot d_{i k} x_{j}+d_{j k} x_{i}-c_{i j} c_{i k} \cdot x_{i} d_{j k}= \\
& =\mathcal{N} \mathcal{D} \mathcal{C}_{i j k} .
\end{aligned}
$$

So, $\mathcal{N} \mathcal{D} \mathcal{C}_{i j k}=0$ in $A$. Hence $N F\left(\mathcal{N D C} \mathcal{C}_{i j k} \mid I\right)=0$ in $T$, and by the previous Lemma $F$ is a Gröbner basis of $I$.

We formalize all the lemmata in the following:

Theorem 2.3 Suppose there is a set $F=\left\{f_{j i} \mid 1 \leq i<j \leq n\right\}$, where

$$
\forall j>i \quad f_{j i}=x_{j} x_{i}-c_{i j} \cdot x_{i} x_{j}-d_{i j}, c_{i j} \in \mathbb{K}^{*}, d_{i j} \in T .
$$

Let the ideal $I=\langle F\rangle \subset T$. If there exists a well-ordering $<$ on $T$, such that $\operatorname{lm}\left(f_{j i}\right)=x_{j} x_{i}$ and $\operatorname{lm}\left(d_{i j}\right)<x_{i} x_{j}$, then the following conditions are equivalent:

1) $F$ is a Gröbner basis for $I$ with respect to $<$,

2) $\forall 1 \leq i<j<k \leq n \quad \mathrm{NF}\left(\mathcal{N D C}_{i j k} \mid F\right)=0$,

3) A $\mathbb{K}$-algebra $A=T / I$ has a Poincaré-Birkhoff-Witt basis.

Remark 2.4 Some historical remarks you can find under Remark 3.5 ,

1. If we assume that $\forall i<j c_{i j}=1$ and $d_{i j}$ are linear polynomials, $\mathcal{N} \mathcal{D} \mathcal{C}_{i j k}$ becomes a famous Jacobi identity ([7]), written in the universal enveloping algebra of a finite dimensional Lie algebra. So, non-degeneracy conditions are generalized Jacobi identities.

2. The equivalence 1) $\Leftrightarrow 3$ ) with several restrictions appeared in [20, [23]; with an assumption that $d_{i j}$ are homogeneous quadratic polynomials it was proved by E. Green in [14] (Th. 2.14).

3. From the proof of the Lemma 2.2 we extract another characterization of PBW property, particularly simple and especially useful for computer algebra systems. Assume that the multiplication $\star$ (from the Lemma) is implemented on $A$ and $\operatorname{lm}\left(d_{i j}\right)<x_{i} x_{j}$. Then we can say whether $A$ has a PBW basis by directly checking, that

$$
\forall 1 \leq i<j<k \leq n \quad\left(x_{k} \star x_{j}\right) \star x_{i}-x_{k} \star\left(x_{j} \star x_{i}\right)=0 .
$$

What happens if we are dealing with an algebra, where non-degeneracy conditions do not vanish? If we consider an algebra, resembling the universal enveloping 
algebra of a finite dimensional Lie algebra but with nonzero non-degeneracy conditions, this will indicate that the underlying algebra, from which the enveloping algebra was built, is not a Lie algebra, since it violates the Jacobi identities.

In general, if the non-degeneracy conditions in the algebra $A=T / I$ ( $T$ and $I$ as before) do not vanish, we observe the following phenomenon - there are more relations than only those of the type $(\star)$, and these hidden relations consist of standard monomials which total degree do not exceed 3. Hence, there exist algebras with no PBW basis but still without zero divisors. We say that the algebra in $n$ variables is degenerate, if it is isomorphic to another algebra, generated by $k<n$ variables.

Example 2.5 Consider the algebra with parameters $q_{1}, q_{2}$,

$$
y x=q_{2} x y+x, \quad z x=q_{1} x z+z, \quad z y=y z .
$$

Here we see that non-degeneracy condition equals $\left(q_{2}-1\right) y z+z$, so it vanishes if and only if there are zero divisors with $\left(\left(q_{2}-1\right) y+1\right) z=0$. So, we have found the hidden defining relation in the algebra, since the Gröbner basis of the ideal $\left\langle y x-q_{2} x y-x, z x-q_{1} x z-z, z y-y z\right\rangle \subset \mathbb{K}\left(q_{1}, q_{2}\right)\langle x, y, z\rangle$ with respect to, say, degree reverse lexicographical ordering, is

$$
G=\left\{y x-q_{2} x y-x, z x-q_{1} x z-z,\left(q_{2}-1\right) z y-z,\left(q_{2}-1\right) y z-z\right\}
$$

or $G^{\prime}=\{y x-x y-x, z\}$, if we assume $q_{2}=1$. In particular, $\mathbb{K}\left(q_{1}, q_{2}\right)\langle x, y, z\rangle /\langle G\rangle$ has a canonical subalgebra, isomorphic to $\mathbb{K}[a, b] /\langle a b\rangle$, hence it has no PBW basis and there are zero divisors. Meanwhile $\mathbb{K}\left(q_{1}\right)\langle x, y, z\rangle /\left\langle G^{\prime}\right\rangle$ degenerates to the algebra $\mathbb{K}\left(q_{1}\right)\langle x, y\rangle /\langle y x-x y-x\rangle$, which is integral but it has a basis $\left\{x^{a} y^{b}\right\}$, though a PBW basis for $\mathbb{K}\left(q_{1}\right)\langle x, y\rangle /\langle y x-x y-x\rangle$ itself, but only a subset of the PBW basis $\left\{x^{a} y^{b} z^{c}\right\}$ for the algebra we were starting with, which is expected from the defining relations.

The first known example of an algebra of that kind with no PBW basis was given in [20] (Example 1.8). It is $B=\mathbb{K}\langle x, y, z \mid y x=x y+x, \quad z x=x z, \quad z y=y z+z\rangle$. Surprisingly, this algebra has a PBW basis (we can check that the non-degeneracy conditions indeed vanish). We believe that there should have been a printing error: the algebra $\mathbb{K}\langle x, y, z \mid y x=x y+x, \quad z x=x z+z, \quad z y=y z\rangle$ from the previous example is pretty close to $B$ and it is degenerate.

\section{Introduction to $G$-algebras}

Now we concentrate on studying the properties of algebras, satisfying the conditions of the Theorem 2.3 .

Take an algebra $A=T / I$ as before. Since it has a PBW basis, we call the elements of this basis monomials of $A$. The set of monomials $\operatorname{Mon}(A)$ could be identified with the $\mathbb{N}^{n}$ by

$$
x^{\alpha}=x_{1}^{\alpha_{1}} x_{2}^{\alpha_{2}} \ldots x_{n}^{\alpha_{n}} \mapsto\left(\alpha_{1}, \alpha_{2}, \ldots, \alpha_{n}\right)=\alpha .
$$

Definition 3.1 Let $<$ be a total well-ordering on $\mathbb{N}^{n}, A$ be a $\mathbb{K}$-algebra with a PBW basis.

1. An ordering $<=<_{A}$ is called a monomial ordering on $A$ if the following conditions hold:

- $\forall \alpha, \beta \in \mathbb{N}^{n} \alpha<\beta \Rightarrow x^{\alpha}<_{A} x^{\beta}$

- $\forall \alpha, \beta, \gamma \in \mathbb{N}^{n}$ such that $x^{\alpha}<_{A} x^{\beta}$ we have $x^{\alpha+\gamma}<_{A} x^{\beta+\gamma}$. 
2. Any $f \in A \backslash\{0\}$ can be written uniquely as $f=c x^{\alpha}+f^{\prime}$, with $c \in \mathbb{K}^{*}$ and $x^{\alpha^{\prime}}<_{A} x^{\alpha}$ for any non-zero term $c^{\prime} x^{\alpha^{\prime}}$ of $f^{\prime}$. We define

$\operatorname{lm}(f)=x^{\alpha}$, the leading monomial of $f$, $\operatorname{lc}(f)=c$, the leading coefficient of $f$, $\operatorname{le}(f)=\alpha, \quad$ the leading exponent of $f$.

Definition 3.2 Let $A=\mathbb{K}\left\langle x_{1}, \ldots, x_{n} \mid f_{j i}=0,1 \leq i<j \leq n\right\rangle$, where

$$
\forall i<j f_{j i}=x_{j} x_{i}-c_{i j} \cdot x_{i} x_{j}-d_{i j}(\underline{x}), c_{i j} \in \mathbb{K}^{*}, d_{i j} \in A .
$$

$A$ is called a $G$-algebra, if the following conditions hold:

- there is a monomial well-ordering $<_{A}$ such that $\forall i<j \operatorname{lm}\left(d_{i j}(\underline{x})\right)<_{A} x_{i} x_{j}$,

- $\forall 1 \leq i<j<k \leq n \mathcal{N D C}_{i j k}=0$ for sets $C=\left\{c_{i j}\right\} \subset \mathbb{K}^{*}$ and $D=\left\{d_{i j}\right\}$.

By the Theorem 2.2 and the construction, any $G$-algebra has a canonical PBW basis $\left\{x_{1}^{\alpha_{1}} x_{2}^{\alpha_{2}} \ldots x_{n}^{\alpha_{n}} \mid \alpha_{k} \geq 0\right\}$. Hence we regard a $G$-algebra (in $n$ variables) as a generalization of a commutative polynomial ring in $n$ variables.

Remark 3.3 Let $A$ be a $G$-algebra. Then, $\forall \alpha, \beta \in \mathbb{N}^{n}$ the leading term of $x^{\alpha} x^{\beta}$ is $c(\alpha, \beta) x^{\alpha+\beta}$ with $c(\alpha, \beta) \in \mathbb{K}^{*}$, hence

$$
\forall f, g \in A \quad \operatorname{lm}(f \cdot g)=\operatorname{lm}(\operatorname{lm}(f) \cdot \operatorname{lm}(g))=\operatorname{lm}(g \cdot f) .
$$

We can rewrite this property also in terms of leading exponents:

$$
\forall f, g \in A \quad \mathrm{le}(f \cdot g)=\mathrm{le}(f)+\mathrm{le}(g) .
$$

Consider now a $\mathbb{K}$-algebra $B$, built on the vector space $\left\{x_{1}^{\alpha_{1}} x_{2}^{\alpha_{2}} \ldots x_{n}^{\alpha_{n}}\right\}$ with such multiplication, that the function le $(\cdot)$ is well-defined on $B$.

Then, if $\forall f, g \in A$ le $(f \cdot g)=\mathrm{le}(f)+\mathrm{le}(g)$, then $B$ is a $G$-algebra.

Definition 3.4 An algebra $A$ is called a Gröbner-ready, or simply a $G R-$ algebra, if there exist an appropriate non-degenerated change of variables $\phi: A \rightarrow$ $A$ and a well-ordering $<_{A}$, such that $\phi(A)$ is either a $G$-algebra or there exist a $G$-algebra $B$ and a proper two-sided ideal $I \subset B$ such that $\phi(A) \cong B / I$.

Remark 3.5 $G$-algebras were first introduced by J. Apel ([1]), however, without requiring the vanishing of non-degeneracy conditions; they were omitted also in the work on PBW algebras (9]), which are defined similarly to $G$-algebras but the presence of PBW basis is required in the definition. In the work [20] on algebras of solvable type authors obtained a criterion for non-degeneracy but did not mention the polynomial conditions $\mathcal{N} \mathcal{D} \mathcal{C}_{i j k}$ explicitly. In [2] and [3] R. Berger introduced $q$-algebras (in our notation, these are the $G$-algebras with the restriction that the polynomials $d_{i j}$ are quadratic), and imposed the vanishing conditions for what he calls " $q$-Jacobi sums" (which coincide with the non-degeneracy conditions) on them. He treated these conditions as quantized Jacobi identities. We have obtained the non-degeneracy conditions independently ([21]) and, moreover, we have shown that the restriction to the quadratic polynomials is not really essential.

It is very natural to study $G$-algebras and their factor-algebras within the same framework. We avoid the name $P B W$-algebras, since in general a factor-algebra of an algebra with a PBW basis does not have PBW basis itself.

Example 3.6 (Examples of $G$-algebras) Quasi-commutative polynomial rings (for example, the quantum plane $y x=q \cdot x y$ ), universal enveloping algebras of finite dimensional Lie algebras, some iterated Ore extensions, some nonstandard quantum deformations ([15], 18]), Weyl algebras and most of various flavors of quantizations 
of Weyl algebras, Witten's deformation of $U\left(\mathfrak{s l}_{2}\right)$, Smith algebras, conformal $\mathfrak{s l}_{2}-$ algebras ([5]), some of diffusion algebras ([17]) and many more.

Remark 3.7 Consider the Sklyanin algebra

$$
S k l_{3}(a, b, c)=k\left\{x_{0}, x_{1}, x_{2}\right\} /\left\langle\left\{a x_{i} x_{i+1}+b x_{i+1} x_{i}+c x_{i+2}^{2} \mid i=1,2,3 \bmod 3\right\}\right\rangle,
$$

where $(a, b, c) \in \mathbb{P}^{2} \backslash F$, for a known finite set $F$. Suppose that $a \neq 0, b \neq 0$. Then we can rewrite the relations in the following way:

$$
x_{1} x_{0}=-\frac{a}{b} x_{0} x_{1}-\frac{c}{b} x_{2}^{2}, \quad x_{2} x_{1}=-\frac{a}{b} x_{1} x_{2}-\frac{c}{b} x_{0}^{2}, \quad x_{2} x_{0}=-\frac{b}{a} x_{0} x_{2}-\frac{c}{a} x_{1}^{2} .
$$

Suppose there is a well-ordering $<$ with $x_{2}<x_{1}<x_{0}$, satisfying the inequalities $x_{2}^{2}<x_{0} x_{1}, x_{0}^{2}<x_{1} x_{2}, x_{1}^{2}<x_{0} x_{2}$. But since then $x_{0} x_{2}^{2}<x_{0}^{2} x_{1}<x_{1}^{2} x_{2}$, we have $x_{0} x_{2}<x_{1}^{2}$, a contradiction to the assumption on $<$ to be a monomial ordering. Hence, unless $c=0$, there is no monomial well-ordering, such that this algebra is a $G$-algebra (If $c=0, S_{k l}(a, b, 0)$ is a quasi-commutative algebra). Note, that nondegeneracy conditions formally vanish on this non- $G$-algebra, hence the ordering condition in the definition 3.2 is essential.

Example 3.8 (Examples of $G R$-algebras) Exterior algebras, Clifford algebras, finite dimensional associative algebras (8]) and more.

\section{Filtrations and properties of $G$-algebras.}

\subsection{Preliminaries.}

Definition 4.1 We recall some definitions explicitly:

1. An algebra $A$ is called filtered, if for every non-negative integer $i$ there is a subspace $A_{i}$ such that

$$
\text { 1) } \left.A_{i} \subseteq A_{j} \text { if } i \leq j, \quad \text { 2) } A_{i} \cdot A_{j} \subseteq A_{i+j} \quad \text { and } 3\right) A=\bigcup_{i=0}^{\infty} A_{i} \text {. }
$$

The set $\left\{A_{i} \mid i \in \mathbb{N}\right\}$ is called a filtration of $A$.

2. An associated graded algebra $\operatorname{Gr}(A)$ of a filtered algebra $A$ is defined to be

$$
\operatorname{Gr}(A)=\bigoplus_{i=1}^{\infty} G_{i} \quad \text { where } G_{i}=A_{i} / A_{i-1} \text { and } A_{-1}=0
$$

with the induced multiplication $\left(a_{i}+A_{i-1}\right)\left(a_{j}+A_{j-1}\right)=a_{i} a_{j}+A_{i+j-1}$.

Theorem 4.2 (Jacobson) Let $A$ be a filtered algebra and $G=\operatorname{Gr}(A)$ be its associated graded algebra. Then

- If $\operatorname{Gr}(A)$ is left (right) noetherian, then $A$ is left (right) noetherian,

- if $\operatorname{Gr}(A)$ has no zero divisors, then $A$ has no zero divisors, too.

Theorem 4.3 (Goldie-Ore) Let $R$ be an integral associative unital ring. If it is left (resp. right) noetherian, then it has a left (resp. right) quotient ring.

Lemma 4.4 Let $Q=\left\{q_{i j} \mid 1 \leq i<j \leq n\right\}$. Consider the transcendental extension $\mathbb{K}(Q)$ of $\mathbb{K}$. A quasi-commutative ring in $n$ variables, associated to the set $Q$ is defined as follows:

$$
\mathbb{K}_{Q}\left[x_{1}, \ldots, x_{n}\right]:=\mathbb{K}(Q)\left\langle x_{1}, \ldots, x_{n} \mid \forall i<j \quad x_{j} x_{i}=q_{i j} x_{i} x_{j}\right\rangle .
$$

Then $\mathbb{K}_{Q}\left[x_{1}, \ldots, x_{n}\right]$ is a noetherian domain. 
Let $A$ be a $G$-algebra. Then we have two different kinds of filtrations on $A$.

4.2 Weighted degree filtration. Let $<_{w}=(<, \bar{w})$ be a weighted degree ordering on $A$, i.e. there is an $n$-tuple of strictly positive weights $\bar{w}=\left(w_{1}, w_{2}, \ldots, w_{n}\right)$ and some ordering $<$ (for example, a (reverse) lexicographical ordering). Then,

$$
\alpha<_{w} \beta \Leftrightarrow \sum_{i=1}^{n} w_{i} a_{i}<\sum_{i=1}^{n} w_{i} b_{i} \text { or, if } \sum_{i=1}^{n} w_{i} a_{i}=\sum_{i=1}^{n} w_{i} b_{i}, \text { then } \alpha<\beta .
$$

Assume that $w_{1} \geq \ldots \geq w_{n}$ and all the weights are positive integers.

Let us define $\operatorname{deg}_{\omega}\left(x^{\alpha}\right):=w_{1} \alpha_{1}+\cdots+w_{n} \alpha_{n}$ and call it a weighted degree

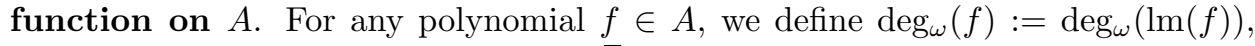
and we note that $\operatorname{deg}_{\omega}\left(x^{\alpha}\right)=0 \Leftrightarrow \alpha=\overline{0}$.

Lemma $4.5 \operatorname{deg}_{\omega}(f g)=\operatorname{deg}_{\omega}(f)+\operatorname{deg}_{\omega}(g)$.

Proof Since on monomials we have

$$
\operatorname{deg}_{\omega}\left(x^{\alpha} x^{\beta}\right)=\operatorname{deg}_{\omega}\left(x^{\alpha+\beta}\right)=\sum_{i=1}^{n} w_{i}\left(\alpha_{i}+\beta_{i}\right)=\operatorname{deg}_{\omega}\left(x^{\alpha}\right)+\operatorname{deg}_{\omega}\left(x^{\beta}\right),
$$

hence, using Remark 3.3

$$
\operatorname{deg}_{\omega}(f g)=\operatorname{deg}_{\omega}(\operatorname{lm}(\operatorname{lm}(f) \operatorname{lm}(g)))=\operatorname{deg}_{\omega}(\operatorname{lm}(f) \cdot \operatorname{lm}(g))=\operatorname{deg}_{\omega}(f)+\operatorname{deg}_{\omega}(g) .
$$

In particular, $\operatorname{deg}_{\omega}(f \cdot g)=\operatorname{deg}_{\omega}(g \cdot f)$.

Let $A_{n}$ be the $\mathbb{K}$-vector space generated by $\left\{m \in \operatorname{Mon}(A) \mid \operatorname{deg}_{\omega}(m) \leq n\right\}$. So, we see that $A_{0}=\mathbb{K}, A_{w_{n}}=\mathbb{K} \oplus \mathbb{K} x_{n}$ if $w_{n-1}>w_{n}$, or $A_{w_{n}}=\mathbb{K} \oplus \bigoplus_{m=1}^{n} \mathbb{K} x_{m}$ if $w_{1}=\ldots=w_{n}$, hence

$$
\forall 0 \leq i<j \quad A_{i} \subseteq A_{j} \subseteq A \text { and } A=\bigcup_{i=1}^{\infty} A_{i} .
$$

From the Lemma 4.5 follows that $\forall 0 \leq i<j \quad A_{i} \cdot A_{j} \subseteq A_{i+j}$. In this case, $G_{i}=A_{i} / A_{i-1}$ is the set of homogeneous elements of degree $i$ in $A$ with $G_{0}=A_{0}=\mathbb{K}$. We have the following:

Lemma 4.6 Suppose we have an algebra $A$, where $\forall i<j \operatorname{deg}_{\omega}\left(d_{i j}\right)<$ $\operatorname{deg}_{\omega}\left(x_{i} x_{j}\right)=w_{i}+w_{j}$. Denote $\bar{x}_{i}=x_{i}+A_{i-1}$. Then

$$
\operatorname{Gr}_{\operatorname{deg}_{\omega}}(A)=\bigoplus_{i=1}^{\infty} G_{i}=\mathbb{K}\left\langle\bar{x}_{1}, \ldots, \bar{x}_{n} \mid \bar{x}_{j} \bar{x}_{i}=c_{i j} \bar{x}_{i} \bar{x}_{j} \forall j>i\right\rangle .
$$

We see that in this case $\operatorname{Gr}_{\operatorname{deg}_{\omega}}(A)$ is isomorphic to the quasi-commutative ring in $n$ variables. Hence, by the Jacobson's Theorem 4.2 $A$ is a noetherian domain.

This Lemma guarantees noetherian and integral properties for Weyl algebras, universal enveloping algebras and some other algebras. Unfortunately, many important algebras (like positively graded quasi-homogeneous algebras) do not satisfy the conditions of the Lemma. But there is another filtration that will do the job. 
4.3 Ordering filtration. Let $<$ be any monomial well-ordering on $A$. For $\alpha \in \mathbb{N}^{n}$, let $A_{\alpha}$ be the $\mathbb{K}$-vector space, spanned by the set $x^{<\alpha} \cup\left\{x^{\alpha}\right\}$, where $x^{<\alpha}:=\left\{x^{\beta} \in A \mid x^{\beta}<x^{\alpha}\right\}$. We see immediately that $A_{\overline{0}}=\mathbb{K}$ and

$$
\forall \beta<\alpha \quad A_{\beta} \subset A_{\alpha} \subset A \text { and } A=\bigcup_{\alpha \in \mathbb{N}^{n}} A_{\alpha} .
$$

The property $A_{\alpha} \cdot A_{\beta} \subseteq A_{\alpha+\beta}$ holds because $\operatorname{lm}\left(x^{\alpha} x^{\beta}\right)=x^{\alpha+\beta}$ (cf. 3.3), hence $A$ is a filtered algebra. Further on, let $\sigma(\alpha):=\max _{<}\{\gamma \mid \gamma<\alpha\}, \sigma(\overline{0})=\emptyset$. Then

$$
\forall \alpha G_{\alpha}=A_{\alpha} / A_{\Sigma(\alpha)}=\left\{x^{\alpha}\right\} .
$$

It follows that

$$
\operatorname{Gr}_{<}(A)=\bigoplus_{\alpha \in \mathbb{N}^{n}} G_{\alpha}=\mathbb{K}\left\langle\bar{x}_{1}, \ldots, \bar{x}_{n} \mid \bar{x}_{j} \bar{x}_{i}=c_{i j} \bar{x}_{i} \bar{x}_{j} \forall j>i\right\rangle,
$$

where $\bar{x}_{i}=x_{i}+A_{\sigma\left(e_{i}\right)}, e_{i}=\left(0, \ldots,{ }_{i}^{1}, \ldots, 0\right)$. So, $\mathrm{Gr}_{<}(A)$ is isomorphic to the quasi-commutative ring in $n$ variables.

Applying the theorems of Jacobson and Goldie-Ore to the result, we get a much more general statement than using just the weighted-degree filtration.

Theorem 4.7 Let $A$ be a $G$-algebra. Then

1) $A$ is left and right noetherian,

2) $A$ is an integral domain,

3) $A$ has a left and right quotient rings.

One can prove 1) also using the PBW Theorem and Dixon's Lemma, like it was done in [10]. Using the structural results from 24] on quasi-commutative rings, we have the following

Proposition 4.8 Let $A$ be a $G$-algebra in $n$ variables. Then

1) the global homological dimension $\operatorname{gl} \cdot \operatorname{dim}(A) \leq n$,

2) the Krull dimension $\operatorname{Kr} \cdot \operatorname{dim}(A) \leq n$,

3) $A$ is Auslander-regular and Cohen-Macaulay algebra.

Remark 4.9 1) and 2) were proved in [9] with the multifiltering technique, which was also applied for the proof of 3) in [11. We proved 1) independently and constructively in 21, using Gröbner bases and our generalization of Schreyer's theorem on syzygies.

Lemma 4.10 There is a category $\mathcal{G} \mathcal{R}$, with $G R$-algebras as objects and $\mathbb{K}$ algebra homomorphisms as morphisms. The category $\mathcal{G} \mathcal{R}$ is closed under the factor operation by two-sided ideals and under the tensor product operation over the ground field $\mathbb{K}$.

Proof Let $A$ (resp. $B$ ) be a $G$-algebra in $n$ (resp. $m$ ) variables with an ordering $<_{A}\left(\right.$ resp. $\left.<_{B}\right): A=\mathbb{K}\left\langle x_{1}, \ldots, x_{n} \mid f_{j i}=0,1 \leq i<j \leq n\right\rangle, B=$ $\mathbb{K}\left\langle y_{1}, \ldots, y_{m} \mid f_{j i}^{\prime}=0,1 \leq i<j \leq m\right\rangle$. Then $C=A \otimes_{\mathbb{K}} B$ is a $G$-algebra in $m+n$ variables with a natural block ordering $\left(<_{A},<_{B}\right)$, since $\forall i, j y_{j} x_{i}=x_{i} y_{j}$ and consequently all non-degeneracy conditions in $C$ vanish. 


\section{Applications of non-degeneracy conditions}

Definition 5.1 Let $A$ be a $G R$-algebra. We call an algebra $A\left(q_{1}, \ldots, q_{m}\right)$, depending on parameters $\left(q_{1}, \ldots, q_{m}\right)$, a $G$-quantization of $A$, if

- $A\left(q_{1}, \ldots, q_{m}\right)$ is a $G R$-algebra for any values of $q_{k}$,

- $A(1, \ldots, 1)=A$.

Let $A$ be a $G$-algebra, generated by $x_{1}, \ldots, x_{n}$.

How to determine the set of all $G$-quantizations of $A$ ?

1. Compute the non-degeneracy conditions and obtain a set $S$ of polynomials in $x_{1}, \ldots, x_{n}$ with coefficients depending on $q_{1}, \ldots, q_{m}$.

2. Form the ideal $I_{S} \in \mathbb{K}\left[q_{1}, \ldots, q_{m}\right]$ generated by all the coefficients of monomials of every polynomial from $S$.

3. Compute the associated primes from the primary decomposition of the radical of $I_{S}$.

4. Throw away every component (that is, an associated prime) which violates $A(1, \ldots, 1)=A$.

Remark 5.2 We use the computer algebra system Singular:Plural [22], with its commutative backbone SINGULAR and noncommutative extension PLURAL. We proceed with the described procedure as follows:

We compute non-degeneracy conditions either with the help of PLURAL or manually. Then, using Singular and its library PRIMDEC [25, we compute the Gröbner basis of $\mathcal{I}$ and then the associated primes of the primary decomposition of the radical of $\mathcal{I}$. Although an implementation of essential algorithm including the primary decomposition is available in polynomial rings over various ground fields $\mathbb{K}$ (like char $\mathbb{K}=0$ or char $\mathbb{K} \gg 0$ as well as their transcendent and simple algebraic extensions), we assume our coefficients $q_{1}, \ldots, q_{m}$ will be specialized in the field $\mathbb{C}$.

Of course, one can insert proprietary criteria and constraints in order to further analyze the set one obtains. Parametric ideals, modules and subalgebras could be studied in a similar way to the investigation of parametric algebras that we present here.

$5.1 G$-quantizations of Weyl algebras. Let $A_{n}=\mathbb{K}\left\langle x_{1}, \ldots, x_{n}, y_{1}, \ldots, y_{n}\right|$ $\left.y_{i} x_{i}=x_{i} y_{i}+1\right\rangle$ be the classical $n$-th Weyl algebra, where we can interpret $y_{i}$ as the differential operator $\partial_{x_{i}}:=\frac{\partial}{\partial x_{i}}$.

From now on, we use the following compact way for encoding the $G$-algebra in 4 variables $\left(c_{i j}, d_{i j}\right.$ are from the Definition 3.2 :

$$
\left(\begin{array}{cccc}
\mathbf{x}_{1} & c_{12} & c_{13} & c_{14} \\
d_{12} & \mathbf{x}_{2} & c_{23} & c_{24} \\
d_{13} & d_{23} & \mathbf{x}_{\mathbf{3}} & c_{34} \\
d_{14} & d_{24} & d_{34} & \mathbf{x}_{\mathbf{4}}
\end{array}\right)
$$

Let's take $A_{2}=\mathbb{K}\left\langle x, \partial_{x}, y, \partial_{y} \mid \partial_{x} x=x \partial_{x}+1, \partial_{y} y=y \partial_{y}+1\right\rangle$. In our new notation it corresponds to the matrix

$$
\left(\begin{array}{cccc}
\mathbf{x} & 1 & 1 & 1 \\
1 & \partial_{\mathbf{x}} & 1 & 1 \\
0 & 0 & \mathbf{y} & 1 \\
0 & 0 & 1 & \partial_{\mathbf{y}}
\end{array}\right)
$$

Note that with such an ordering of variables the PBW basis is $\left\{x^{n_{1}} \partial_{x}^{n_{2}} y^{n_{3}} \partial_{y}^{n_{4}}\right\}$. 
We specify the following constraints to be fulfilled:

- let the general $G$-quantization be $\Delta\left(A_{2}\right)=\Delta\left(A_{2},\left\{c_{i j}\right\},\left\{d_{i j}\right\},<\right)=$ $\mathbb{K}\left\langle x_{1}, x_{2}, x_{3}, x_{4} \mid x_{j} x_{i}=c_{i j} x_{i} x_{j}+d_{i j}, \forall j>i\right\rangle$

- $\forall i<j \quad c_{i j} \in \mathbb{K}^{*}, d_{i j} \in \mathbb{K}$ (as for $d_{i j}$, we consider two cases only: $d_{i j}=0$ and $d_{i j} \neq 0$ ). It means we investigate only "linear" $G$-quantizations.

- $d_{12}=d_{34}=1$

Since $\forall i<j d_{i j} \in \mathbb{K}$, for any well-ordering $<$ on $\Delta\left(A_{2}\right)$ we have $d_{i j}<x_{i} x_{j}$ and $\Delta\left(A_{2}\right)$ is a $G$-algebra in 4 variables, if non-degeneracy conditions vanish. However, if we choose $<$ to be a well-ordering, $\Delta\left(A_{2}\right)$ does not depend on the concrete one. In our encoding it looks the following way:

$$
\left(\begin{array}{cccc}
\mathbf{x} & c_{12} & c_{13} & c_{14} \\
1 & \partial_{\mathbf{x}} & c_{23} & c_{24} \\
d_{13} & d_{23} & \mathbf{y} & c_{34} \\
d_{14} & d_{24} & 1 & \partial_{\mathbf{y}}
\end{array}\right)
$$

Since the set $\mathcal{U}_{3}=\{(i, j, k) \mid 1 \leq i<j<k \leq 4\}$ in this case is equal to $\{(1,2,3),(1,2,4),(1,3,4),(2,3,4)\}$, we have four equations derived from the four non-degeneracy conditions which $\forall(i, j, k) \in \mathcal{U}_{3}$ look as follows:

$$
d_{i j}\left(c_{i k} c_{j k}-1\right) \cdot x_{k}+d_{i k}\left(c_{j k}-c_{i j}\right) \cdot x_{j}+d_{j k}\left(1-c_{i j} c_{i k}\right) \cdot x_{i} .
$$

Now we define two sets of commutative variables $C=\left\{c_{i j} \mid 1 \leq i<j \leq 4\right\}$ and $D=\left\{d_{i j} \mid 1 \leq i<j \leq 4\right\} \backslash\left\{d_{12}, d_{34}\right\}$ (since $d_{12}=d_{34}=1$ ). Then we have the following ideal in the commutative polynomial ring $\mathbb{K}[C, D]$ in 10 variables,

$$
\mathcal{I}=\left\langle d_{i j}\left(c_{i k} c_{j k}-1\right), d_{i k}\left(c_{j k}-c_{i j}\right), d_{j k}\left(c_{i j} c_{i k}-1\right) \mid(i, j, k) \in \mathcal{U}_{3}\right\rangle .
$$

Using a computer algebra system Singular (12]), we compute the Gröbner basis of $\mathcal{I}$ and then the primary decomposition of the radical of $\mathcal{I}$ ([25]). Performing the computations, we find out, that the 4 -dimensional variety, defined by $\sqrt{\mathcal{I}}$, consists of 8 components (corresponding to associated prime ideals). Let us denote the corresponding types of algebras by $\Delta_{1}, \ldots, \Delta_{8}$. Now we list them all, using the following considerations:

- $d_{i j}$ : if there are no restrictions on some $d_{i j}$, we depict it by $*$ in the matrix, interpreting it as a free ("random") parameter,

- $c_{i j}$ : if no conditions on some $c_{i j}$ are given, we will introduce the parameters $q^{\prime}, q^{\prime \prime}$ for "single" (appearing only once) coefficients and $q$ for "block" (appearing more than once) coefficients in the corresponding matrix. These parameters are viewed then as the generators of the transcendental field extension $\mathbb{K}(\underline{q})$.

$$
\begin{array}{rr}
\Delta_{1}=\left(\begin{array}{cccc}
\mathbf{x} & 1 & 1 & 1 \\
1 & \partial_{\mathbf{x}} & 1 & 1 \\
* & * & \mathbf{y} & 1 \\
* & * & 1 & \partial_{\mathbf{y}}
\end{array}\right), \quad \Delta_{2}=\left(\begin{array}{cccc}
\mathbf{x} & -1 & -1 & -1 \\
1 & \partial_{\mathbf{x}} & -1 & -1 \\
* & * & \mathbf{y} & -1 \\
* & * & 1 & \partial_{\mathbf{y}}
\end{array}\right), \\
\Delta_{3}=\Delta_{3}\left(q^{\prime}\right)=\left(\begin{array}{cccc}
\mathbf{x} & q^{\prime} & 1 & 1 \\
1 & \partial_{\mathbf{x}} & 1 & 1 \\
0 & 0 & \mathbf{y} & 1 \\
* & 0 & 1 & \partial_{\mathbf{y}}
\end{array}\right), \quad \Delta_{4}=\Delta_{4}\left(q^{\prime}\right)=\left(\begin{array}{cccc}
\mathbf{x} & q^{\prime} & -1 & -1 \\
1 & \partial_{\mathbf{x}} & -1 & -1 \\
0 & 0 & \mathbf{y} & -1 \\
* & 0 & 1 & \partial_{\mathbf{y}}
\end{array}\right),
\end{array}
$$




$$
\begin{aligned}
& \Delta_{5}\left(q^{\prime}, q^{\prime \prime}, q\right)=\left(\begin{array}{cccc}
\mathbf{x} & q^{\prime} & q & q^{-1} \\
1 & \partial_{\mathbf{x}} & q^{-1} & q \\
0 & 0 & \mathbf{y} & q^{\prime \prime} \\
0 & 0 & 1 & \partial_{\mathbf{y}}
\end{array}\right), \quad \Delta_{6}=\Delta_{6}(q)=\left(\begin{array}{cccc}
\mathbf{x} & q & q^{-1} & q \\
1 & \partial_{\mathbf{x}} & q & q^{-1} \\
0 & * & \mathbf{y} & q \\
0 & 0 & 1 & \partial_{\mathbf{y}}
\end{array}\right), \\
& \Delta_{7}=\Delta_{7}(q)=\left(\begin{array}{cccc}
\mathbf{x} & q & q^{-1} & q \\
1 & \partial_{\mathbf{x}} & q & q^{-1} \\
* & 0 & \mathbf{y} & q^{-1} \\
0 & 0 & 1 & \partial_{\mathbf{y}}
\end{array}\right), \quad \Delta_{8}=\Delta_{8}(q)=\left(\begin{array}{cccc}
\mathbf{x} & q & q & q^{-1} \\
1 & \partial_{\mathbf{x}} & q^{-1} & q \\
0 & * & \mathbf{y} & q^{-1} \\
0 & 0 & 1 & \partial_{\mathbf{y}}
\end{array}\right) .
\end{aligned}
$$

Now we check, whether $\Delta_{i}(1, \ldots, 1)=A$. Using the encoding it turns to be especially simple - all the $G$-quantization of $A$ could be represented by the $\Delta_{5}\left(q^{\prime}, q^{\prime \prime}, q\right)$, since, substituting everywhere the free parameter $*$ with 0 , we have

$$
\begin{gathered}
\Delta_{1}=\Delta_{5}(1,1,1), \quad \Delta_{3}=\Delta_{5}\left(q^{\prime}, 1,1\right), \quad \Delta_{6}=\Delta_{5}\left(q, q, q^{-1}\right), \\
\Delta_{7}=\Delta_{5}\left(q, q^{-1}, q^{-1}\right), \quad \Delta_{8}=\Delta_{5}\left(q, q^{-1}, q\right) .
\end{gathered}
$$

If we substitute $*$ with a unit, the only $G$-quantization of $A$ is $\Delta_{5}\left(q^{\prime}, q^{\prime \prime}, q\right)$. Note, that in any case $\Delta_{2}$ and $\Delta_{4}$ are not $G$-quantizations.

It's interesting to see how this classification reflects some of classical algebras related to $A_{2}$. Recall the encodings of algebras:

$$
A_{1} \sim\left(\begin{array}{cc}
\mathbf{x} & 1 \\
1 & \partial_{\mathbf{x}}
\end{array}\right), \quad A_{1}(q) \sim\left(\begin{array}{cc}
\mathbf{x} & q \\
1 & \partial_{\mathbf{x}}
\end{array}\right)
$$

Then it's easy to see that

- $A_{2}=A_{1} \otimes_{\mathbb{K}} A_{1}$ is of the type $\Delta_{1}$,

- $A_{1}\left(q^{\prime}\right) \otimes_{\mathbb{K}\left(q^{\prime}\right)} A_{1}$ is of the type $\Delta_{3}$,

- $A_{1}\left(q^{\prime}\right) \otimes_{\mathbb{K}\left(q^{\prime}, q^{\prime \prime}\right)} A_{1}\left(q^{\prime \prime}\right)$ is of the type $\Delta_{5}\left(q^{\prime}, q^{\prime \prime}, 1\right)$.

What happens to $\Delta_{3}, \Delta_{6}, \Delta_{7}, \Delta_{8}$, if we substitute the free parameter with some unit? They are not $G$-quantizations anymore, but still interesting $G$-algebras, like $\Delta_{7}$ where $*$ is replaced with 1 : we get an algebra with $\partial_{x}, \partial_{y}$ acting as classical differentials on $x, y$ (which generate $A_{1}\left(q^{-1}\right)=\mathbb{K}\left\langle x, y \mid y x=q^{-1} x y+1\right\rangle$ ).

In order to go back to the classical PBW basis $\left\{x^{n_{1}} y^{n_{2}} \partial_{x}^{n_{3}} \partial_{y}^{n_{4}}\right\}$ it is enough just to change our encoding, permuting the corresponding entries:

$$
\left(\begin{array}{cccc}
\mathbf{x} & q^{\prime} & q & q^{-1} \\
1 & \partial_{\mathbf{x}} & q^{-1} & q \\
0 & 0 & \mathbf{y} & q^{\prime \prime} \\
0 & 0 & 1 & \partial_{\mathbf{y}}
\end{array}\right) \longrightarrow\left(\begin{array}{cccc}
\mathbf{x} & q & q^{\prime} & q^{-1} \\
0 & \mathbf{y} & q & q^{\prime \prime} \\
1 & 0 & \partial_{\mathbf{x}} & q \\
0 & 1 & 0 & \partial_{\mathbf{y}}
\end{array}\right)
$$

The $G$-quantization of the type $\Delta:=\Delta_{5}$ could be generalized for higher Weyl algebras.

Theorem 5.3 Consider $A_{n}=\mathbb{K}\left\langle x_{1}, \ldots, x_{n}, \partial_{x_{1}}, \ldots, \partial_{x_{n}} \mid \partial_{x_{i}} x_{i}=x_{i} \partial_{x_{i}}+1\right\rangle$. Given $n$ "single" parameters $p_{1}, \ldots, p_{n}$ and $m=\frac{1}{2} n(n-1)$ "block" parameters $q_{1}, \ldots, q_{m}$, then there exists a $m+n$-parameter $G$-quantization $\Delta_{n}(\underline{p}, \underline{q})$ which has the following form in the compact encoding: 


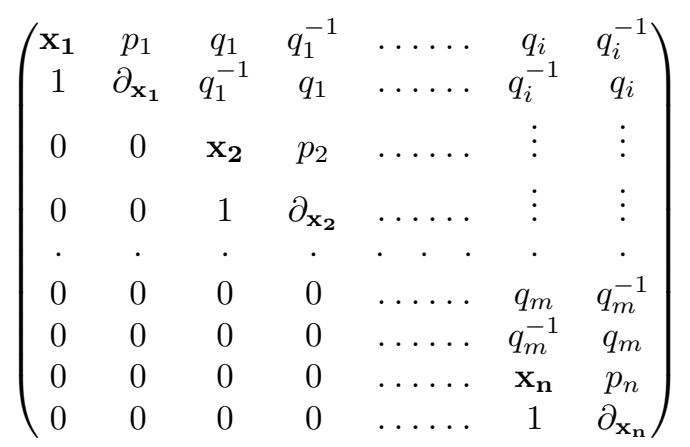

We count in such a way that in the matrix above $i=\frac{1}{2}(n-1)(n-2)+1, n \geq 2$. This deformation has the following properties:

1. $\forall n \geq 1 \Delta_{n}(\underline{p}, \underline{q})$ is a simple noetherian domain with the PBW basis

$$
\left\{x_{1}^{\alpha_{1}} \partial_{x_{1}}^{\alpha_{n+1}} \ldots x_{n}^{\alpha_{n}} \partial_{x_{n}}^{\alpha_{2 n}} \mid \alpha \in \mathbb{N}^{2 n}\right\},
$$

which can be easily rewritten to the classical PBW basis.

2. Let $1 \leq s<m$ and $\nu(k):=\frac{1}{2} k(k-1)$.

Define the index set

$I=\bigoplus_{t=0}^{m-s-1} I_{t}$, where $I_{t}=\{\nu(s+t)+1, \ldots, \nu(s+t+1)-t\} \forall 0 \leq t \leq m-s-1$.

Set $q^{\prime}:=\left\{q_{i} \mid i \in I\right\}$ and $\underline{q^{\prime \prime}}:=\left\{q_{i} \mid \nu(s+1)+1 \leq i \leq m\right\} \backslash \underline{q^{\prime}}$. Then

$\underline{q^{\prime}}=\underline{1} \Rightarrow \Delta_{n}(\underline{p}, \underline{q})=\Delta_{s}\left(p_{1}, \ldots, p_{s} ; q_{1}, \ldots, q_{\nu(s)}\right) \otimes_{\mathbb{K}\left(\underline{p}, \underline{q} \backslash \underline{q^{\prime}}\right)} \Delta_{m-s}\left(p_{s+1}, \ldots, p_{n} ; \underline{q^{\prime \prime}}\right)$.

In particular,

$$
\begin{gathered}
\underline{q^{\prime}}:=\left(q_{i}, \ldots, q_{m}\right)=\underline{1} \Rightarrow \Delta_{n}(\underline{p}, \underline{q})=\Delta_{n-1}\left(\underline{p} \backslash p_{n}, \underline{q} \backslash \underline{q^{\prime}}\right) \otimes_{\mathbb{K}(\underline{p})} A_{1}\left(p_{n}\right), \\
\underline{q}=\underline{1} \Rightarrow \Delta_{n}(\underline{p}, \underline{q})=A_{1}\left(p_{1}\right) \otimes_{\mathbb{K}(\underline{p})} \cdots \otimes_{\mathbb{K}(\underline{p})} A_{1}\left(p_{n}\right), \\
\underline{q}=\underline{1}, \underline{p}=\underline{1} \Rightarrow \Delta_{n}(\underline{p}, \underline{q})=A_{n}=\bigotimes_{i=1}^{n} A_{1} .
\end{gathered}
$$

Proof We have to show that $\Delta$ is a $G$-algebra. It becomes clear from the definition we have to show only that the non-degeneracy conditions vanish. We do it by induction on $n . \Delta_{1}\left(p_{1}\right)$ is a $q$-Weyl algebra $A_{1}\left(p_{1}\right)$, and the theorem is obviously true for it. Now assume that $\Delta_{n-1}$ is a $G$-algebra. We construct $\Delta_{n}$ from $\Delta_{n-1}$ with a single parameter $p_{n}$ and $n-1$ block parameters $q_{i}, \ldots, q_{m}$. We have to show that the following equalities hold:

$$
\begin{aligned}
\partial_{x_{n}} *\left(y_{k} * y_{l}\right)- & \left(\partial_{x_{n}} * y_{k}\right) * y_{l}=0, \quad x_{n} *\left(y_{k} * y_{l}\right)-\left(x_{n} * y_{k}\right) * y_{l}=0, \\
& \partial_{x_{n}} *\left(x_{n} * y_{k}\right)-\left(\partial_{x_{n}} * x_{n}\right) * y_{k}=0 .
\end{aligned}
$$

where $\left(y_{k}, y_{l}\right)$ are pairs of generators of $\Delta_{n-1}$ with $y_{k}>y_{l}$ and $*$ is the multiplication on $\Delta$. In fact it suffices to show that $\forall 1 \leq k<n$

$$
\begin{gathered}
\partial_{x_{n}} *\left(\partial_{x_{k}} * x_{k}\right)-\left(\partial_{x_{n}} * \partial_{x_{k}}\right) * x_{k}=0, \\
x_{n} *\left(\partial_{x_{k}} * x_{k}\right)-\left(x_{n} * \partial_{x_{k}}\right) * x_{k}=0, \\
\partial_{x_{n}} *\left(x_{n} * x_{k}\right)-\left(\partial_{x_{n}} * x_{n}\right) * x_{k}=0, \\
\partial_{x_{n}} *\left(x_{n} * \partial_{x_{k}}\right)-\left(\partial_{x_{n}} * x_{n}\right) * \partial_{x_{k}}=0 .
\end{gathered}
$$


Let us prove the first equality (the other will follow analogously):

$\partial_{x_{n}} *\left(\partial_{x_{k}} * x_{k}\right)=\partial_{x_{n}} *\left(p_{k} x_{k} \partial_{x_{k}}+1\right)=p_{k} q_{i+k-1}^{-1} x_{k} \partial_{x_{n}} \partial_{x_{k}}+\partial_{x_{n}}=p_{k} x_{k} \partial_{x_{k}} \partial_{x_{n}}+\partial_{x_{n}}$, $\left(\partial_{x_{n}} * \partial_{x_{k}}\right) * x_{k}=q_{i+k-1}^{-1} \partial_{x_{k}}\left(\partial_{x_{n}} * x_{k}\right)=q_{i+k-1} \partial_{x_{k}} q_{i+k-1}^{-1} x_{k} \partial_{x_{n}}=p_{k} x_{k} \partial_{x_{k}} \partial_{x_{n}}+\partial_{x_{n}}$, where $i=\frac{1}{2}(n-1)(n-2)+1$. The claim follows.

The properties of $\Delta$ one can read directly from the encoding we use.

5.2 Quadratic algebras in 3 variables. Consider a class of $G$-algebras in $n$ variables which relations are homogeneous of degree 2 . We call these algebras quadratic $G$-algebras. Let us have a look at the case when $n=3$.

Assume that the relations are given in terms of non-deformed commutators (i.e. $\left.c_{i j}=1 \forall j>i\right)$. Let us fix an ordering, say, Dp (degree lexicographical ordering) with $x>y>z$. Then the relations of quadratic algebra $A$, satisfying the ordering condition from the definition of $G$-algebras, should be of the following form:

$$
\begin{aligned}
y x & =x y+a_{1} x z+a_{2} y^{2}+a_{3} y z+\xi_{1} z^{2}, \\
z x & =x z+\xi_{2} y^{2}+a_{5} y z+a_{6} z^{2}, \\
z y & =y z+a_{4} z^{2} .
\end{aligned}
$$

Computing the non-degeneracy conditions, we construct the ideal

$$
\mathcal{I}=\left\langle 2 a_{2} a_{4}+a_{1} a_{5}-a_{4} a_{5}, 2 a_{2} a_{4}^{2}-a_{4}^{2} a_{5}+a_{1} a_{6}+a_{3} a_{4}\right\rangle .
$$

We see that the non-degeneracy conditions do not depend on $\xi_{1}, \xi_{2}$, so we are working further on within the ring $\mathbb{K}\left[a_{1}, \ldots, a_{6}\right]$. Moreover, the ideal $\mathcal{I}$ is a radical ideal. Computing the primary decomposition, we get two associated prime ideals $\mathcal{I}_{1}=\left\langle 2 a_{2} a_{4}+a_{1} a_{5}-a_{4} a_{5}, a_{1} a_{5}^{2}-a_{3} a_{5}+2 a_{2} a_{6}-a_{5} a_{6}, a_{1} a_{4} a_{5}-a_{3} a_{4}-a_{1} a_{6}\right\rangle$ and $\mathcal{I}_{2}=\left\langle a_{1}, a_{4}\right\rangle$, corresponding to components $\mathcal{V}_{1}$ and $\mathcal{V}_{2}$ of the 4-dimensional variety $\mathcal{V}(\mathcal{I})=\mathcal{V}_{1} \cup \mathcal{V}_{2}$

Let us start with the component $\mathcal{V}_{2}$. Since $a_{1}=a_{4}=0$ on it, consider the subalgebra $H=\mathbb{K}\langle y, z \mid z y=y z\rangle$. In fact we can call the algebra "solvable", since then $[H, x] \in H,[A, A]=H$ and $[[A, A], A]=0$. So, the component $\mathcal{V}_{2}$ gives us the family of "solvable" algebras, depending on six random parameters $a_{2}, a_{3}, a_{5}, a_{6}, \xi_{1}, \xi_{2}$ having the following relations:

$$
y x=x y+a_{2} y^{2}+a_{3} y z+\xi_{1} z^{2}, \quad z x=x z+\xi_{2} y^{2}+a_{5} y z+a_{6} z^{2}, \quad z y=y z .
$$

We use the decomposition $\mathcal{V}(\mathcal{I})=\mathcal{V}_{2} \cup \mathcal{V}_{1}=\mathcal{V}_{2} \oplus\left(\mathcal{V}_{1} \backslash \mathcal{V}_{2}\right)$. On the latter set the parameters are algebraically dependent, so we can express, for example, $a_{2}, a_{6}$ :

$$
a_{2}=\frac{1}{2}\left(1-\frac{a_{1}}{a_{4}}\right) a_{5}, \quad a_{6}=a_{4}\left(a_{5}-\frac{a_{3}}{a_{1}}\right) .
$$

We see, that the family of algebras, arising from $\mathcal{V}_{1} \backslash \mathcal{V}_{2}$ depend on two nonzero parameters (here $a_{1}, a_{4}$ ) and four random parameters (here $a_{3}, a_{5}, \xi_{1}, \xi_{2}$ ).

5.3 Diffusion algebras. A diffusion algebra ([17]) is generated by $\left\{D_{i}, 1 \leq i \leq n\right\}$ over $\mathbb{K}$ subject to the relations:

$$
c_{i j} D_{i} D_{j}-c_{j i} D_{j} D_{i}=x_{j} D_{i}-x_{i} D_{j}, \forall i<j
$$

where $c_{i j} \geq 0$ and $x_{i}$ are coefficients from the field.

We will assume that $\forall i, j c_{i j}>0$ and therefore concentrate on analyzing the $G$-algebras among the diffusion algebras (the authors of the article [17] studied all the possible diffusion algebras). 
For the diffusion algebras we compute the non-degeneracy conditions for a fixed triple $(i, j, k)$ in a similar way as we $\operatorname{did}$ for $G$-algebras. After computing the primary decomposition, we get eight components and we do the classification of algebras, following the approach from [17. Each component of the primary decomposition corresponds to a different form of algebra. One component corresponds to the type $\mathbf{A}$, three to the type $\mathbf{B}$, three to the type $\mathbf{C}$ and one component to that of $\mathbf{D}$.

A type : every $x_{m}$ is nonzero. Then there are relations

$c_{j k}=c_{k i}=c_{i k}=c_{j i}=c_{i j}=c_{k j}$, that is, we obtain universal enveloping algebras of Lie algebras with relations

$$
\left[D_{i}, D_{j}\right]=\frac{x_{j}}{c_{i j}} D_{i}-\frac{x_{i}}{c_{i j}} D_{j} .
$$

B type : one of $x_{m}$ is equal to zero. In the case $x_{i}=0$, we have

$$
c_{k i}=c_{i j}, c_{i k}-c_{k i}=c_{j k}-c_{k j}=c_{j i}-c_{i j}=: c
$$

And the relations are the following:

$$
\begin{aligned}
c_{i j} D_{i} D_{j}-\left(c_{i j}+c\right) D_{j} D_{i} & =x_{j} D_{i}, \\
c_{j k} D_{j} D_{k}-\left(c_{j k}-c\right) D_{k} D_{j} & =x_{k} D_{j}-x_{j} D_{k}, \\
\left(c_{i j}+c\right) D_{i} D_{k}-c_{i j} D_{k} D_{i} & =x_{k} D_{i} .
\end{aligned}
$$

The cases $x_{j}=0$ and $x_{k}=0$ could be handled in an analogous way.

C type : one of $x_{m}$ is nonzero. Let $x_{j}=0, x_{k}=0$. Then $c_{i j}-c_{j i}=c_{i k}-c_{k i}=: c$, and there are relations

$$
\begin{aligned}
c_{i j} D_{i} D_{j}-\left(c_{i j}-c\right) D_{j} D_{i} & =-x_{i} D_{j}, \\
c_{j k} D_{j} D_{k}-c_{k j} D_{k} D_{j} & =0, \\
c_{i k} D_{i} D_{k}-\left(c_{i k}-c\right) D_{k} D_{i} & =-x_{i} D_{k} .
\end{aligned}
$$

The cases $x_{i}=x_{k}=0$ and $x_{i}=x_{j}=0$ could be done analogously.

D type : every $x_{m}$ is equal to zero. There are no additional constraints on $c_{i j}$ and it is not surprising, since this type consists of quasi-commutative algebras with relations

$$
D_{l} D_{m}=\frac{c_{m l}}{c_{l m}} D_{m} D_{l}, \quad(l, m) \in\{(i, j),(i, k),(j, k)\}
$$

As we can see, we obtained the same classification of $G$-algebras among the diffusion algebras as in [17. The advantage of our approach lies in the automation of the process, in particular, we can consider more variables and build the classification, using the computer algebra methods only. Thus, the proposed approach is limited only by the overall performance of the computing facilities.

\section{Concluding remarks}

We have shown the nature of non-degeneracy conditions and their interplay with the PBW basis property in a wide class of algebras. Through combining both noncommutative and commutative computational methods we are able to classify families of parametric algebras having PBW basis and being noetherian domains.

In the work [19], H. Kredel introduced a generalization of algebras of solvable type. He considered rings, which do not necessarily commute with the ground 
field but the corresponding relations are compatible with monomial orderings. In such rings the existence of PBW basis is interesting question, partially answered by H. Kredel. Another objects of interest are iterated Ore extensions (many rings of solvable type could be presented like such extensions), where the classical way of construction does not guarantee the non-degeneracy. It is quite interesting to investigate these rings for further generalization of non-degeneracy conditions and for the existence of theorems, analogous to the Theorem 2.3. There are several works by T. Gateva-Ivanova on skew polynomial rings with binomial relations, where noetherian and other important properties of such rings were studied with the help of Gröbner bases.

We created a computer algebra system Singular:PluRAL ([21]), a kernel extension of a well-known in the commutative world system Singular ([12]). Thus we have the same user programming language, common libraries, help system etc. It turns out to be very useful while performing the computations like in the previous chapter, that is, when we need combined (i.e. both commutative and noncommutative) applications of Gröbner bases at the same time. As far as we know, SinGULAR:PLURAL is the only modern system allowing user to do such combined computations. Main computational objects in the system are $G R$-algebras, where we can compute Gröbner bases for left and two-sided ideals and for left modules, modules of syzygies, free resolutions, intersections with subalgebras and many more (see [21] or 22 for description of all these algorithms). We put a lot of efforts in implementing the algorithms as efficient as possible and the latest tests indicate that the performance of Singular:Plural is quite good. You can find detailed information on Singular:Plural either in 22] or at http://www.singular.uni-kl.de/plural.

\section{References}

[1] Apel, J.: Gröbnerbasen in nichtkommutativen Algebren und Ihre Anwendung, Dissertation, Universität Leipzig, 1988.

[2] Berger, R.: The quantum Poincaré-Birkhoff-Witt theorem. Commun. Math. Phys. 143, No. 2, 215-234 (1992).

[3] Berger, R.: Quantification de l'identité de Jacobi. (Quantization of the Jacobi identity). C. R. Acad. Sci., Paris, Sér. I 312, No.10, 721-724 (1991).

[4] Bergman, G.: The diamond lemma for ring theory. Adv. Math. 29, 178-218 (1978).

[5] Benkart, G.; Roby, T.: Down-up algebras, J. Algebra 209, No. 1, 305-344 (1998).

[6] Ringel, C. M. PBW-bases of quantum groups, J. Reine Angew. Math. 470, 51-88 (1996).

[7] Dixmier, J.: Enveloping Algebras. AMS, Providence, RA, 1996.

[8] Drozd, Y.; Kirichenko, V.: Finite dimensional algebras. With an appendix by Vlastimil Dlab. Translated from Russian by Vlastimil Dlab, Berlin, Springer Verlag, 1994.

[9] Gomez-Torrecillas, J.; Lobillo, F.J.: Global homological dimension of multifiltered rings and quantized enveloping algebras. J. Algebra 225, No. 2, 522-533 (2000).

[10] Bueso, J. L.; Gómez Torrecillas, J.; Lobillo, F.J.; Castro, F.J.: An introduction to effective calculus in quantum groups, in Caenepeel, S. and Verschoren, A. (eds.), Rings, Hopf algebras, and Brauer groups, Marcel Dekker, New York, 55-83, (1998).

[11] Gomez-Torrecillas, J.; Lobillo, F.J.: Auslander-regular and Cohen-Macaulay quantum groups, arXiv:math.RA/0104283, 2001.

[12] G.-M. Greuel, G. Pfister, with contributions by O. Bachmann, C. Lossen and H. Schönemann, "A SINGULAR Introduction to Commutative Algebra", Springer Verlag, 2002.

[13] Green, E. Multiplicative Bases, Gröbner Bases, and Right Gröbner Bases, Journal of Symbolic Computation 29 No. 4/5, 2000.

[14] Green, E. Noncommutative Groebner bases, and projective resolutions. Draexler, P. (ed.), Computational methods for representations of groups and algebras. Proc. of the Euroconference in Essen, Germany, April 1-5, 1997. Basel: Birkhaeuser. Prog. Math. 173, 29-60 (1999). 
[15] Havlicek, M.; Klimyk, A.; Posta, S.: Central elements of the algebras $U_{q}^{\prime}\left(\mathrm{so}_{m}\right)$ and $U_{q}^{\prime}\left(\mathrm{iso}_{m}\right)$, arXiv. math. QA/9911130.

[16] Li, H. Noncommutative Gröbner bases and filtered-graded transfer. Springer, 2002.

[17] Isaev, A.; Pyatov, P.; Rittenberg, V.: Diffusion algebras, arXiv.math.QA/0103603.

[18] Iorgov, N.: On the Center of q-Deformed Algebra $U_{q}^{\prime}\left(\mathrm{so}_{3}\right)$ Related to Quantum Gravity at $q$ a Root of 1, in Proc. IV Int. Conf. "Symmetry in Nonlinear Mathematical Physics", Kyiv, Ukraine, 2001.

[19] Kredel, H.: Solvable polynomial rings. Aachen, Shaker (1993).

[20] Kandri-Rody, A.; Weispfenning, V.: Non-commutative Gröbner bases in algebras of solvable type, Journal of Symbolic Computation 9, No.1, 1-26 (1990).

[21] Levandovskyy, V.: On Gröbner bases for noncommutative G-algebras, in H. Kredel, W. K. Seiler (eds.) Proc. of the 8th Rhine Workshop on Computer Algebra, Mannheim, Germany, 2002.

[22] Levandovskyy V.; Schönemann, H. Plural - a computer algebra system for noncommutative polynomial algebras. In Proc. of the International Symposium on Symbolic and Algebraic Computation (ISSAC'03), ACM Press (2003).

[23] Mora, T.: An introduction to commutative and noncommutative Gröbner bases, Theor. Comput. Sci. 134, No.1, 131-173 (1994).

[24] McConnell, J.C. and Robson, J.C. Noncommutative Noetherian rings. With the cooperation of L. W. Small. Providence, AMS (2001).

[25] W. Decker, G. Pfister, and H. Schönemann. primdec.lib. A Singular 2.0 library for computing the primary decomposition and radical of ideals (2001). 